\title{
An Intellectual Energy Device for Household Appliances Using Artificial Neural Network
}

\author{
Hariprasath Manoharan, ${ }^{1}$ Yuvaraja Teekaraman $\left(D^{\circ},{ }^{2}\right.$ Ramya Kuppusamy, ${ }^{3}$ \\ and Arun Radhakrishnan (iD) ${ }^{4}$ \\ ${ }^{1}$ Department of Electronics and Communication Engineering, Panimalar Institute of Technology, Chennai 600 123, India \\ ${ }^{2}$ Mobility, Logistics, and Automotive Technology Research Centre, Faculty of Engineering, Vrije Universiteit Brussel, \\ Brussel 1050, Belgium \\ ${ }^{3}$ Department of Electrical and Electronics Engineering, Sri Sairam College of Engineering, Bangalore City 562 106, India \\ ${ }^{4}$ Department of Electrical \& Computer Engineering, Jimma Institute of Technology, Jimma University, Jimma, Ethiopia
}

Correspondence should be addressed to Yuvaraja Teekaraman; yuvarajastr@ieee.org and Arun Radhakrishnan; arun.radhakriahnan@ju.edu.et

Received 30 September 2021; Accepted 6 November 2021; Published 19 November 2021

Academic Editor: Vijay Kumar

Copyright (c) 2021 Hariprasath Manoharan et al. This is an open access article distributed under the Creative Commons Attribution License, which permits unrestricted use, distribution, and reproduction in any medium, provided the original work is properly cited.

\begin{abstract}
This article highlights the importance of implementing intelligent monitoring devices with the internet of things (IoT) for observing the amount of charges on different appliances in each household. In India, it has been observed that $20 \%$ of power is wasted due to commercial appliances where the amount of charge flow is much excess to corresponding appliances. Therefore, to perceive information about the flow of charges, it is necessary to implement an intelligent device, and it is possible to obtain exact information on the flow of charges with the help of wireless sensor networks (WSN). Even most of the researchers have developed an intelligent device for monitoring the amount of charges but delay, energy consumption, and cost of implementation are much higher. It is always necessary to extract precise information at corresponding time periods for reducing the delay in packet transmission of a specific network. To excerpt such real-time data in the network layer, an active procedure should be followed by integrating dissimilar network areas inside a single cluster, and binary coded artificial neural network (BCANN) is introduced to acquire information about hidden layers. To prove the effect of such integration process, several tests have been prepared using online and offline analyses where simulation results prove to be much effective in case of all different scenarios to an extent of $52.4 \%$ when compared to existing methods.
\end{abstract}

\section{Energy Depletion with Sensors}

In current generation network operations, creation has been moving towards an inordinate inclination that guarantees financial improvement in terms of energy management and conversion. Even with the inclusion of all technological components, capacity of data dissemination will vary after the existence of such energy conversion process. In addition, for this type of real-time energy-saving, information about all buildings should be integrated where only authorized users are specified for operating the entire network. If the emission of different fumes is present within web applications, then it is necessary to implement a standard technique for managing indicators worldwide. Furthermore, all home appliances should be monitored using a wireless device approach, which can be accessed in a remote medium. For all the abovementioned indicators, the solution can be provided only by implanting sensors, which stand as basic fundamental for controlling energy transactions in physical layers. These types of sensors are created not only to save energy but also to prevent unbalance in load quantities with proper system installation and preservations. Therefore, any change in electrical components will be instantaneously monitored in the network where a predesign can be made with such effective process. 
1.1. Literature: A Brief Review. A lot of researchers have analyzed the impact of the flow of charges with IoT technology, but only few researchers succeeded in exploring the precise method, where flow of charges is found appropriately in each household. A smart compact energy meter is designed with IoT-based technology where all demand has been monitored for the purpose of site management in construction buildings [1]. These categories of demand management will have a separate pattern for consuming power in many areas, but if separate patterns are present, then the designed cluster will vary with environmental conditions with zero energy-saving capability. Even a discrete energy model is designed for saving energy with an optimized model where data will be disseminated across the entire network [2]. This type of technology is generated recently, but the major disadvantage is no routing approach is supported in the network layer that makes the processing customs of a packet to be less significant. The authors in [3] have established a technical description that will even be implemented in all public buildings for reducing energy consumption. The process of data transfer is also established with IoT process for even small buildings to build a smart city. But the flow of charges can be monitored using such technical description, and electricity consumption in every household cannot be controlled at any stage. However, a vigorous environmental condition can be obtained at much higher efficiency [3]. Also, the aforementioned method [3] is the only enhancement of the energy management information system where central data can be gathered from all buildings. Even big data collection is also reported, thus making feature extraction on consuming energy for making significant decisions in all public areas. For achieving high quality of service, a neural network is implemented by using hidden layers where all observed errors in data processing using IoT will be automatically corrected with three-layer process, which includes a value replacement technique. By implementing a value replacement technique, all variables will be reduced with outer elimination, but such techniques require a high cost of implementation [3]. Therefore, to reduce the cost of implementing, a wireless device that monitors electricity consumption using Wi-Fi technology is implemented [4].

Implementation of such a technique in households will help each individual analyze the amount of charges that are consumed on both weekly and monthly basis. In addition, a survey has been reported for monitoring consumer behavior, where actual power consumption will be monitored by installed sensors. By incorporating such a technique, any illicit activities can be easily reported to the control center, thus preventing excessive loss of charges [4]. Whenever any user gives a request to the control center, then a database system will be completely searched, and as a result, the amount of consumed energy will be reported to the user. The integrated method that is reported in [4] is pertinent to all appliances, and it can be monitored separately. If any appliance is using more charges, then that particular appliance will be switched off for a particular period of time, and even it can be adjusted as per the user's wish. This method saves more energy, but if IoT is integrated into all appliances with the same characteristics, then it is a very challenging task for reporting all necessary data to the control center, and it is a time-consuming process [4]. Both the authors of reference $[3,5]$ have discussed various issues related to inappropriate installation and remedies to overcome those issues but [4] focused on identifying the inopportune behavior of each appliance that is present in each household. Therefore, for monitoring the abovementioned issue, a real-time data is needed with submetering changes. Thus, the changes are clearly observed using IoT where redundant charges will be permitted to move in another gateway. Even modified counsels are provided to each user if the rates of flow of charges are much lesser. But if more number of nodes is needed, then it is difficult to transfer the charges in other meters, and this method will provide more errors than expected [4]. Therefore, to reduce the error and provide clean energy throughout the cities, a digital method is needed that reduces energy wastage [6]. To incorporate this energy-saving method, a computational intelligence with IoT is introduced. This type of intelligent method converts the traditional networks to intelligent power networks. However, the digital method fails to provide local optimized solutions if the flow of charges is higher in certain areas. Also, managing big data for entire transmission networks is much time-consuming since computational complexity is much higher [6].

Before converting a traditional system to a digitized system, it is much important to calculate each electrical quantity [7]. If appropriate awareness of all electrical quantities is known, then oscillation in charges can be controlled manually. Once manual process prospers, then an IoT technology can be implemented for analyzing the power at various households. After this way of automatic conversion, any information change in electrical quantity will be sent via the application platform. Therefore, the method will be time-consuming, and there is a need to control only essential quantity instead of all quantity [7]. But the method will create misperception in case if electrical quantity is assorted. To reduce the cost of implementation further, more renewable energy sources must be considered with optimization technique [8] where information about charges can be obtained by integrating renewable energy sources. There will be a huge growth in the energy sector only when renewable source is added with IoT technology. If the segment of renewable energy is higher, then consumption of energy will be lesser due to lower impact on environmental conditions. Moreover, an efficient energy processing technique will be provided with the transformation from centralized systems to smart systems [8]. Conversely, such a smart method requires a green IoT technology where the cost of implementation will be much higher and data storage techniques will be provided with less security concern.

Furthermore, analyses have been carried out by considering the effect of load forecasting with machine learning algorithms [9]. If load forecasting is analyzed in a short period with intelligent algorithms, then smart grid operation will be more effective in predicting energy management. For validating the method of load forecasting, an Arduino UNO processor has been implemented for acquiring the load data. 
But more advanced processors have been developed with more memory capacity that is used for storing the data at a low cost [9]. When Arduino UNO is used, then three different stages of online forecasting will be distributed that consist of a sensual system, microcontroller unit, and data analytics stage. In these three stages, more variables should be combined according to different requirements, thus making the process to be more complex [9]. In addition to load forecasting, a demand response (DR) program with price-based strategy is examined [10]. For effective functioning of DR, a bacterial foraging algorithm has been preferred where scheduled operation in monitoring the power management has been made. When DR is introduced, then electricity consumption will be much lower with more efficient utilization of resources [10]. An automatic response will be created with IoT technology for a smart grid environment at a minimized cost. But in DR, automatic monitoring and controlling of each appliance in terms of different electrical quantities is a complex process.

For smart grid operations, speed of monitoring and data transfer process should be higher [11]. Therefore, for achieving a higher speed of operation, more number of ports should be enabled at a high security rate. Also, operations on distributed computing need to be evaluated as a long-term process, and for such long-term continuity, energy consumption should be reduced with high quality of service [11]. But the speed of the data transfer process does not guarantee the proper operation of the smart grid in energy sectors. It is also observed that any eccentricity in solar power can be monitored by remote users, and even small tunings can be made regularly by users at the control center [12]. When solar cells are enabled, then it is necessary to use illuminate sensors to monitor the degree of charges that is passed through each cell. This process will provide more improvement in the energy sector only when IoT is enabled in its corresponding infrastructure [12]. In imminent processing techniques, real-time applications using solar cells with IoT will have a much higher impact, thus providing promising consumption on the electricity market. If a solar plant is installed, then a computer-aided system is needed for monitoring the photovoltaic cells with web-based nodes [13]. When fifth-generation networks are updated then data communication for uploading base data for monitoring, the smart grid environment in each household will be different [14]. Therefore, more number of constraints need to be framed with the low-cost optimization process, which will be provided with opportunistic allocation. But direct updating to fifth-generation networks with low variables is a much composite process [14].

1.2. Research Gap. There are many predominant gaps that can be observed in preceding works [1-14], where the researchers have not integrated an IoT-based smart intelligence system using sensors for monitoring the energy, which is represented by charges. Even no elucidation has been provided in recent works about the consumption of energy in home appliances where existing works have indicated that usage of solar power will reduce the amount of energy than expected. Even it is acceptable that solar panels can be integrated, but the cost of implementing such devices will be much higher, and to overcome this, cost-effective technology should be developed in an effective manner. These identified gaps are deciphered using proposed technology, which is explained in the subsequent subsection.

1.3. Proposed Methodology. To overcome the abovementioned breach, an automatic monitoring system has been introduced in the proposed work. Also, the main motivation behind this awareness is to check both failure and high passing of charges, which is one of the most dangerous problems in all households. Therefore, for avoiding this most dangerous problem, an intelligent device has been integrated, where all changes in the passing of charges to each household can be monitored at remote locations by the control center. By implementing IoT technology with neural networks, even the hidden layer problem in the identification of charges will be solved, and all household applications can be easily replaced using sensing devices within a short period of time at a low cost.

1.4. Objectives. To solve all the difficulties that are present during transitory of charges in each appliance, the authors have framed a multiobjective model for the proposed work as follows:

(i) To implement IoT with sensing process for testing the amount of charges that fluctuates with marginal index values

(ii) To minimize the oscillated values of all appliances in each household for increasing the lifetime of the network

(iii) To reduce the delay of the communication process by reducing peak ratio values

(iv) To limit the consumption of installed nodes according to changes in environs

(v) To reduce the total cost of installation

For attaining all the aforementioned objectives, an optimization algorithm, which is termed as binary coded artificial neural network (BCANN), is integrated to observe the changes appropriately and monitor each household in an efficient way. In addition, for integrating the intelligent monitoring device, a dynamic management protocol is used, where energy consumption of nodes will be reduced.

\section{Problem Formulation}

It is necessary that proper amount of electricity should be consumed at all houses, and it is possible to monitor the correct amount by the flow of charges. This flow of charge is defined as an electrical quantity, and for the corresponding quantity, two different parameters should be considered such as current and time. Therefore, these two basic parameters can be defined using the following equation:

$$
C_{i}=I_{i} \times t_{i},
$$


where $C_{i}$ represents the charge that is passing through crosssectional areas in all houses and is expressed in Columbus, $I_{i}$ indicates the current consumed in each house and is expressed in Amperes, and $t_{i}$ represents different time period, which is observed for 24 hours.

Equation (1) indicates the basic formulation of charges to all appliances. But the proposed method will integrate the process of IoT for monitoring different adaptable appliances, which include heater, air conditioners, induction appliances, and so on. Therefore, if a high amount of charges pass through cross-sectional areas, then it should be reported to the corresponding individual in each house. Therefore, in the proposed method, the appliances are divided into three different types, which are expressed in the following equation:

$$
U_{i}=U_{c}(i)+U_{p}(i)+U_{a}(i)
$$

where $U_{i}$ represents the total number of electrical utensils in the house, $U_{c}(i)$ denotes control equipment that needs to be measured from $\mathrm{ON}$ position, $U_{p}(i)$ signifies time period of critical equipment that is measured between $\mathrm{ON}$ and $\mathrm{OFF}$ position, and $U_{a}(i)$ indicates dire equipment that is measured at $\mathrm{ON}$ position.

In addition, the remaining time period of other electrical equipment will also be monitored to find the accurate consumption of electricity without any wastage. The aforementioned parameters will be indicated using either 0 or 1 . If 1 is indicated, then the equipment is very critical as more charges are passing through it for different time periods. But if 0 is indicated, then electrical equipment is free from the flow of charges, or a very low amount of charges pass through it where no external control is required. Therefore, depending on the status indicator, both restrained consumption and tangible consumption will be calculated using the following equation:

$$
\text { marginal energy index }=\frac{r c_{i}-t c_{i}}{r c_{i}} \times 100,
$$

where $r c_{i}$ indicates energy that is measured at the user end and $t c_{i}$ represents actual energy that is predefined according to installed apparatus.

It is definite that to achieve marginal index, the appliances should be switched from high to low state. This option is always defined by the user with certain precautions. Therefore, average ratios should be calculated by using energy parameters, which can be expressed as follows:

$$
\operatorname{PAR}(i)=\min \frac{U_{c}(i)+U_{a}(i)}{\text { total energy }},
$$

where $\operatorname{PAR}(i)$ represents the peak to average ratio in the instance where power is controlled.

The amount of average power, which is represented in equation (4), should be calculated properly in order to level all corresponding loads. Once the load level becomes equal; then in automatic process, cost of consumed energy will become lesser, thus achieving a higher lifetime to all electrical equipment. If the users are consuming power at a scheduled time period, then delay will occur during the control process, which is defined using the following equation:

$$
d_{i}=\min \left(U_{p}(i)+U_{a}(i)\right)
$$

where $d_{i}$ represents the total delay that is deliberated at a scheduled time.

Equation (5) indicates that if the time period of both critical and noncritical equipment are minimized, then the minimum delay will be attained. If the time period is more during scheduled operation, then the equipment will face the worst condition as a number of charges in cross-sectional areas will become higher. Therefore, it is essential to define the limits of probable energy consumption using the number of cycles as given in the following equation:

$$
\mathrm{TC}_{i}=n w_{i} \times \text { total energy, }
$$

where $T C_{i}$ represents the total cycle that is obtained at $i^{\text {th }}$ iteration and $n w_{i}$ denotes the number of cycles that a household is getting in weekly analysis.

The constraint in equation (6) is that it can be applied only to appliances that are matching with minimum standards. If the number of weekly cycles is considered, then it is necessary to determine the average weight of each appliance. But in the proposed method, a baseline value will be assumed for the automatic operation, which provides more advantage when compared to other methods. Once the appliance exceeds the baseline value, then automatic replacement with necessary components will be made. Therefore, the total cost of monitoring with replacement can be formulated as in the following equation:

$$
\operatorname{cost}_{i}=\min \sum_{i=1}^{n} \sum_{p=1}^{24}\left(\left(U_{i}, T C_{i}\right) \times \rho(i),\right.
$$

where $\rho(i)$ represents the daily charge for convention and replacement.

Equation (7) is formulated as an optimization problem by minimizing the total number of electric utensils with the number of cycles. Both minimized values will be multiplied with daily charges for calculating the total cost. Therefore, the proposed method can be formulated as a minimization problem, which is represented using equation (8).

$$
Z(i)=\min \left(\operatorname{cost}_{i}, \mathrm{TC}_{i}, U_{i}, d_{i}\right) .
$$

If the abovementioned parameters in equation (8) are minimized, then demand for energy will be reduced with the help of smart monitoring systems.

\section{Optimization Algorithm}

For accumulating data from several households, an intelligent optimization algorithm with hidden layers should be integrated. The major reason for using a hidden layer approach is that the proposed method is nonlinear, and data from previous prototypes can be easily obtained. Therefore, an intelligent machine learning algorithm, which is termed as binary coded artificial neural network (BCANN) is integrated [15]. As mentioned earlier, the status of the data 
collection process will be indicated by binary numbers $\{0,1\}$ with one hidden layer. The primary advantage of BCANN is that if any error is present in data, then it can be corrected at the output. Also, the number of variables used in preprocessing system will be reduced, thus obtaining a cluster. If a cluster is formed, then the number of errors can be reduced with dynamic monitoring of charges in every household. During this prediction process, the error should be much closer to a measured value, which is calculated using the following equation:

$$
\delta(i)=\frac{\sum_{i=1}^{n}\left(y_{i}-\widehat{y}_{i}\right)\left(y_{i}^{\prime}-\widehat{y_{i}^{\prime}}\right)}{\sum_{i=1}^{n} \sqrt{\left(y_{i}-\widehat{y}_{i}\right)\left(y_{i}^{\prime}-\widehat{y_{i}^{\prime}}\right)}}
$$

where $y_{i}$ and $\widehat{y}_{i}$ represents the measured and predicted energy (charges) consumption from each household, respectively, and $y_{i}^{i}$ and $\widehat{y_{i}^{\prime}}$ denotes the average measured and predicted energy (charges) consumption from each household, respectively.

The resultant value of $\delta(i)$ will be processed to the next step for visualizing the data using an advanced application process. The accuracy of the resultant correlated value will be much higher, and it is computed using the following equation:

$$
\Delta_{i}=\frac{100}{\text { observed samples }} \sum_{i=1}^{n} \frac{\left(y_{i}-\widehat{y}_{i}\right)\left(y_{i}^{\prime}-\widehat{y_{i}^{\prime}}\right)}{\left(y_{i}-\widehat{y_{i}}\right)\left(y_{i}^{\prime}-\widehat{y_{i}^{\prime}}\right)}
$$

where $\Delta_{i}$ denotes the accuracy of $i^{\text {th }}$ samples that is distributed with the number of observed samples.

The loop will be simulated until the error is minimized. For the simplification process, the number of iterations is assumed to be 500; therefore, within 500 iterations, accurate results will be obtained, and the status will be displayed at the end. This type of process is termed cross-corroboration that indicates that data will be normalized at each cluster region, and at the final stage, if any increase in error is observed, then total iteration will be stopped [16]. The flow chart of BCANN is given in Figure 1.

\subsection{Dynamic Management Protocol for Energy Consumption.} Since wireless sensor nodes are attached to each household, it is necessary to manage the energy in terms of both charges and sensors. Therefore, a dynamic protocol is needed for managing the energy consumption during two different stages that include operation and training stages [17]. In the operation stage, the main nodes will broadcast all information and will send a separate ID for deploying all information at the receiver side. Similarly, in the training stage, the main nodes will check all locations of each household and will divide the households as a cluster within network ranges. If a particular house falls apart from network coverage, then a separate cluster will be formed, and this cluster formation provides more advantages for management purposes. This type of cluster

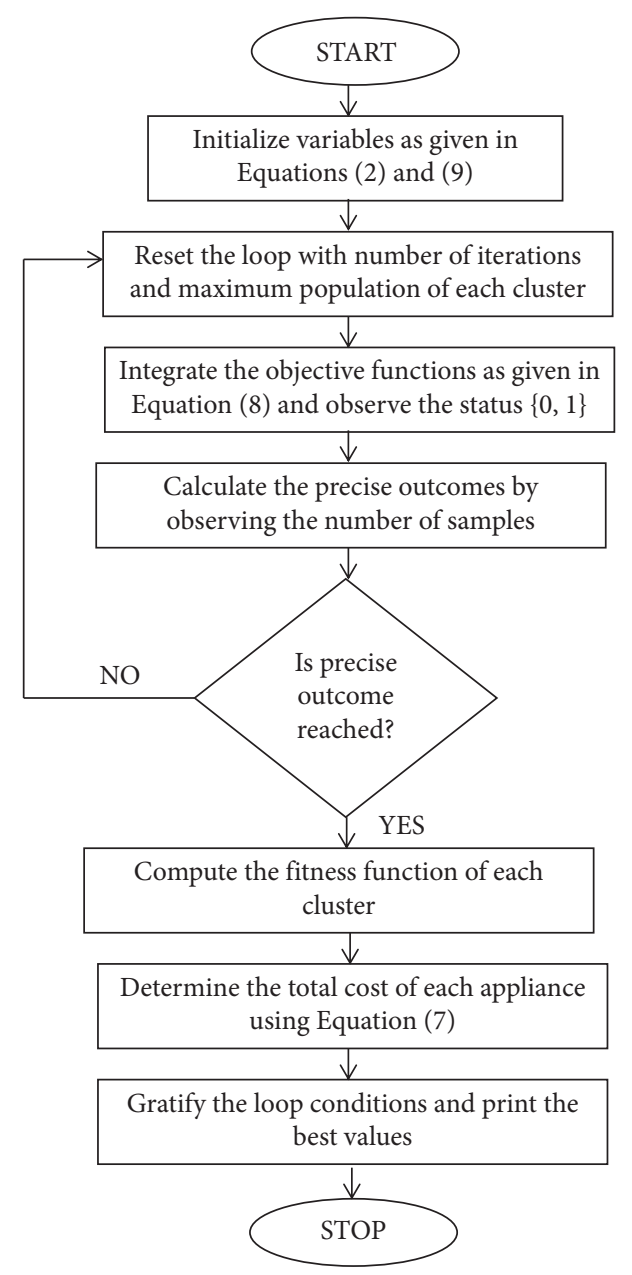

Figure 1: Flow chart of energy consumption using BCANN.

formation is termed snoop and alters, where depending on availability of packets information will be passed continuously. Therefore, the radius of the cluster should be higher, and it can be calculated using the following equation:

$$
r(i)=\sqrt{\frac{n(i)}{\pi}},
$$

where $n(i)$ denotes the number of households.

Equation (11) indicates that for cluster formation, the number of households should be divided by $\pi$, and the square root of the radius will form a snoop state at the operation stage. This forms a new methodology for monitoring households at the control center where information can be passed at early stages with less delay. The proposed dynamic management protocol differs from existing protocols in the way of the proper organization where clusters will be formed at the exact position. This forms the advantage primarily for monitoring all appliances in each household. If dynamic management protocol is integrated with BCANN, then performance will be better at all hidden nodes with efficient energy and less delay. 


\section{Results and Discussion}

In this section for the projected formulations, the observed values by sensors using neural networks have been simulated using MATLAB. Since monitoring is carried out with online estimation, the simulation analysis is plotted in MATLAB. In the first step of the projected technique on energy consumption in each household, the charge for total and individual appliances needs to be evaluated, and the resultant values will be used for evaluating the following scenarios.

Scenario 1: examination on marginal energy indices

Scenario 2: control of PAR

Scenario 3: observation of delay

Scenario 4: calculation of overall cost

Scenario 5: variation of error rate

The aforementioned scenarios will be observed based on case studies with charges observed in a week for each distinct appliance. The simulation setup for the proposed method is based on different layers, which are used for analyzing big data and processing techniques using intelligent algorithms. The proposed method is used for function analytics with fewer variables in each household.

4.1. Case 1. In this scenario, the basic evaluation on charges that are observed in both day and night is projected. It is definite that charges that are passing through each appliance will have more effect on energy consumption. Also, different appliances will be turned $\mathrm{ON}$ at each time, thus allowing the users to save their energy. Figure 2 shows the observed charges in both day and night, which are denoted in Amperes. The values are simulated for a period of 6 days in a continuous manner where the average values are reserved for 100 households. It can also be observed in Figure 2 that more amount of energy is consumed only during nighttimes. But in real time, the charges passing through both day- and nighttimes will be approximately equal. Due to the existence of LED lights, the charges consumed at each household are lesser at nighttimes, and it has been reported that smart appliances will provide more savings on energy when compared to standard appliances. Therefore, for better saving, it is opined to use smart appliances, and it will save more charges that are passing at nighttimes.

It can be observed in Figure 2 that during daytime, the maximum amount of charges that are consumed is 87,000 Amperes. Whereas in nighttime, it is observed to higher that is equal to 92,000 Amperes. By using this, basic evaluation charges on the separate appliance are observed in scenario 2 .

4.2. Case 2. In this scenario, charges that are observed for specific appliance during night- and daytime are calculated. In the proposed method, for better understanding, running of motor that is considered as critical appliance during daytime and air conditioners as critical appliance during nighttime in each household are deliberated. For restrained empathetic, refrigerator that is switched $\mathrm{ON}$ at day and nighttimes is also considered. The same technique is

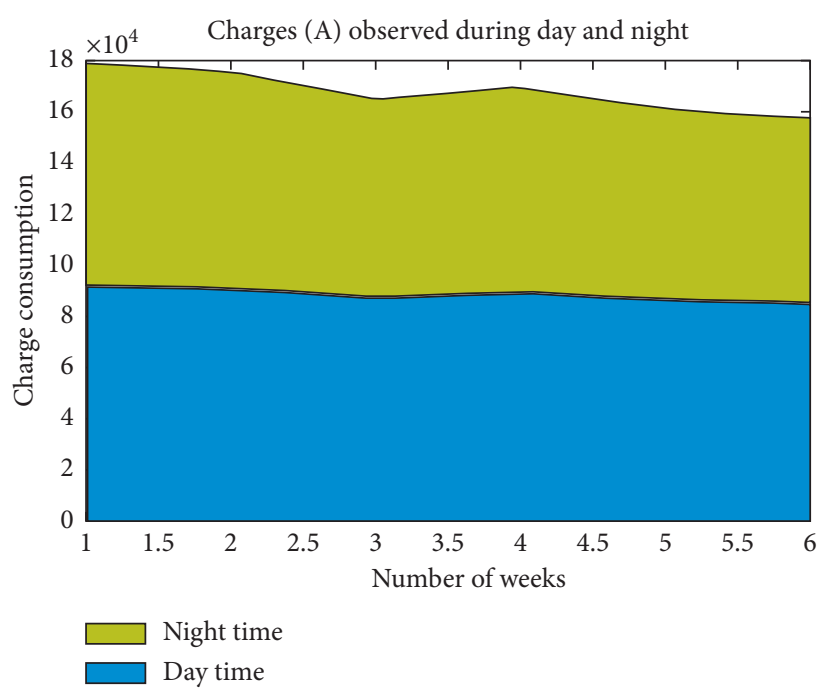

FIGURE 2: Diversity of charges during day and night.

evaluated for a period of 6 days by considering an average of 100 households. Figure 3 shows the charges that are observed for each critical appliance that are used at day- and nighttimes. Similar to scenario 1, it is perceived that sensors are integrated at each appliance, and all are connected directly to an online monitoring system. Any abnormal changes in transitory of charges will make the particular appliance to be switched OFF for safety conditions, thus saving more energy.

4.3. Scenario 1. In this scenario, marginal stability index that is required for each appliance to control the outlay is calculated. If the amount of charges that are passed to a certain appliance turns back to its original point, then the system can be labeled to be in the normal state. If an appliance turns back to its normal state within a certain period of time or within the allowable limit, then expenditure can be controlled, and more energy can be saved. The marginal stability for the proposed method is calculated by determining two different energy values, where one value will be a predetermined form by the manufacturer and the other will be observed by the user. If the appliance crosses the marginal stability, then expenditure for users will be much higher, and a distinct warning condition will also be placed inside the intelligent devices. Figure 4 shows the marginal energy index for all appliances in different households. The total marginal index for an average of 100 households is calculated with tangible consumption. From Figure 4, it can be observed that the marginal index for the proposed method is lesser because a timely warning about the expenditure of each appliance is provided. This scenario proves the importance of IoT and the need for data processing techniques in energy sectors.

From Figure 4, it can be observed that the proposed method produces a less marginal index for a maximum of 20 different appliances. For example, if the number of appliances are 10, then expenditure produced by the existing method [3] is 3.56 lakhs, whereas for the proposed method it is 3.17 lakhs. Thus, the proposed method using the neural 


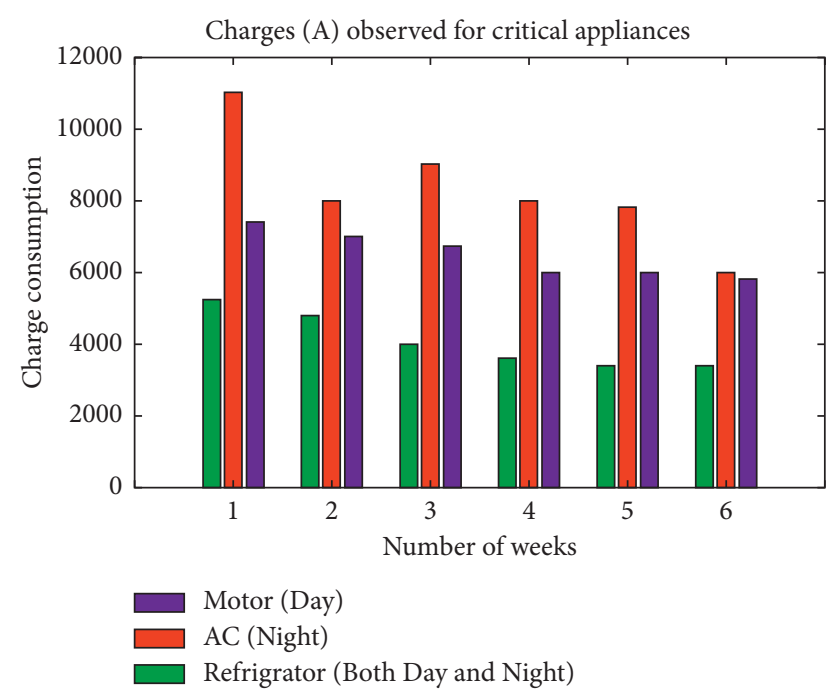

FIGURE 3: Diversity of charges for critical appliances.

network can be applied effectively to all appliances in households.

4.4. Scenario 2. In this scenario, peak to average ratio (PAR) for different time periods is observed for all appliances that are operating both day and night. The change in values has been detected for a time interval of three hours, which starts from 12:00 a.m. In the proposed method, instant charges that are passed at a certain time interval are compared with average charges, and they are recompensed if more high changes are observed. Figure 5 shows the simulated value of PAR that is varied from 12:00 a.m. to 21:00 p.m. for different households.

It can be seen from Figure 5 that the PAR for the proposed neural network method is very lesser, or in other terms, the PAR is compensated to average values as expected. For example, at 9:00 a.m., the original PAR is $38 \mathrm{~dB}$, but at the same time, the proposed method achieves low PAR, which is equal to $34 \mathrm{~dB}$. Therefore, if an IoT technology is integrated for data processing, then peak charges that are used for each appliance can be controlled regularly, and it can be tracked for different time periods as per operator requirement. The proposed method reduces the waiting time of all appliances since PAR is much lesser but even though some sort of delay exists. The amount of delay that is produced by proposed and existing methods is discussed in Scenario 5.

4.5. Scenario 3. Since IoT technology is enabled, it is necessary to check the amount of delay in addition to waiting time. Therefore, the aforementioned three different appliances which include AC, refrigerator, and motor are taken under consideration and their delays in producing charges after varying PAR are observed. Figure 6 deliberates delay in hours for different appliances where if the charges are higher, then a delay period should be provided for passing the charge to other appliances so that the corresponding appliance will not have any overload effect. In the traditional

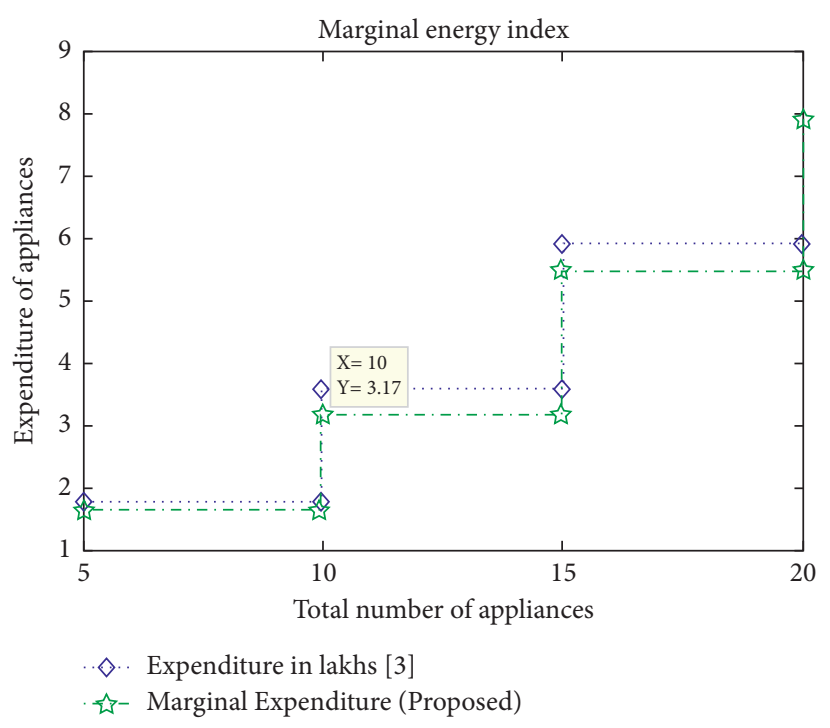

FIgURE 4: Diversity of charges for critical appliances.

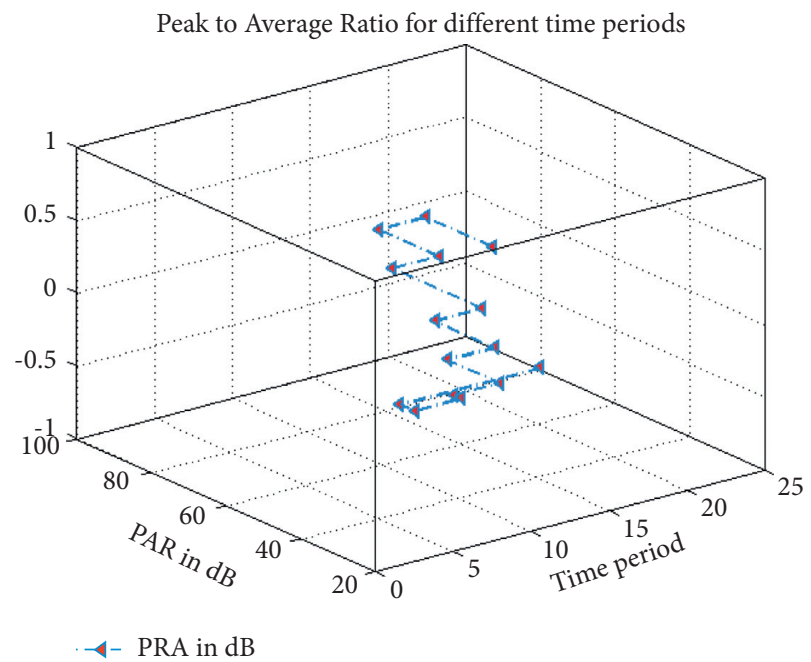

FIgURE 5: Observed PAR for different appliances in each household.

method, if a circuit experiences overload, then the total appliance should be switched OFF for some period of time. This process is much complex where each individual will be using different appliances, but in the proposed method, delay will be clearly indicated to each individual so that the automatic switching of different appliances will be divided according to the flow of charges. This method will also provide more advantages to all industries where more appliances are operated.

4.6. Scenario 4. In this scenario, total cost of installing IoT technology with essential sensors for all appliances in different households is calculated and simulated. Total cost is calculated as given in equation (7) where daily charges for replacement and convention should be minimized. If daily charges are much higher, then the data processing technique needs to be installed in an additional manner. Therefore, 


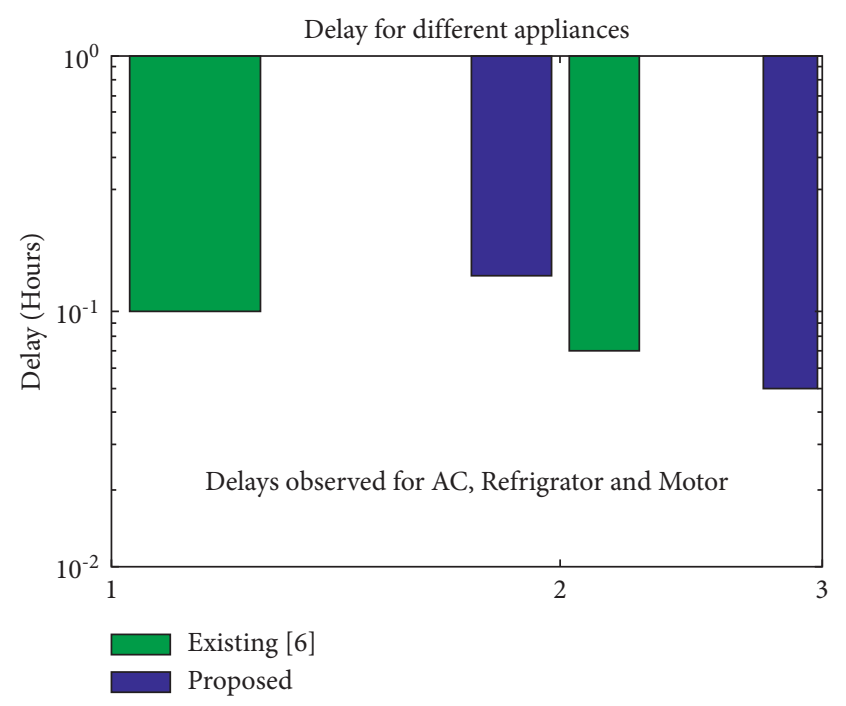

Figure 6: Delay for different appliances.

such a state demands a higher cost of installation and that particular case is avoided in the proposed method. Figure 7 shows the simulated values in terms of cost, which is expressed in lakhs.

From Figure 8, it can be observed that for the same installation, the proposed method produces low cost when compared with the existing method [4]. For example, if the number of nodes is 1,000 , then the total cost of installing IoT for each appliance with energy consumption and daily charges will be 46.9 lakhs, whereas with same number of nodes, but with different devices, the installation cost for the existing method cost is 53.5 lakhs, which is much higher than proposed method. Therefore, scenario 4 proves that at a low cost of installation, the proposed method performs well by monitoring appliances at each household.

4.7. Scenario 5. In this scenario, variation in measured traversed energy is calculated and is indicated decibels that are varied with respect to the number of time periods in seconds. The intention of such measures in variation is that suddenly, there may be voltage drop that is present across the designed path, and to overcome such designed paths, errors in variations are observed. Even the same situation can be measured by considering the amplitude of several events that have happened after switching on necessary home appliances. But the abovementioned measures are possible when the IoT process is not present. Since the proposed method integrated IoT process, measurable events are different where only irresistible devices are considered without conversion of energy. Further changes in error measurement can be measured even in hourly duration, but in the proposed method, a constant change in the time period is provided, and the simulated error rate is deliberated in Figure 7.

From Figure 7, it is experimental that the error rate that varies with respect to signals is very lesser for proposed method, whereas for the existing method, it is much higher, and it starts with 10,000 decibels. Such huge variations are

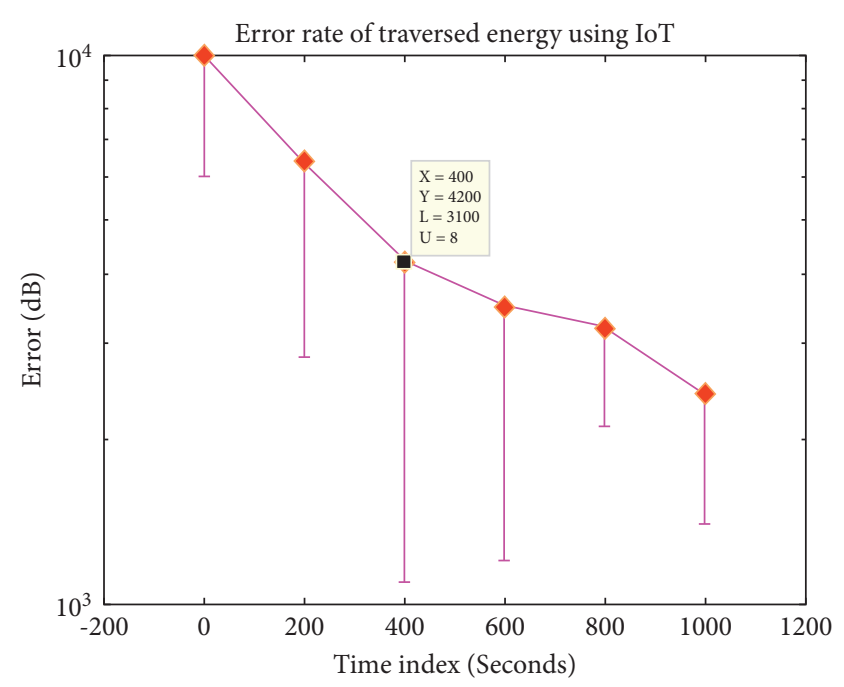

FIgURE 7: Signal error rate for traversed energy.

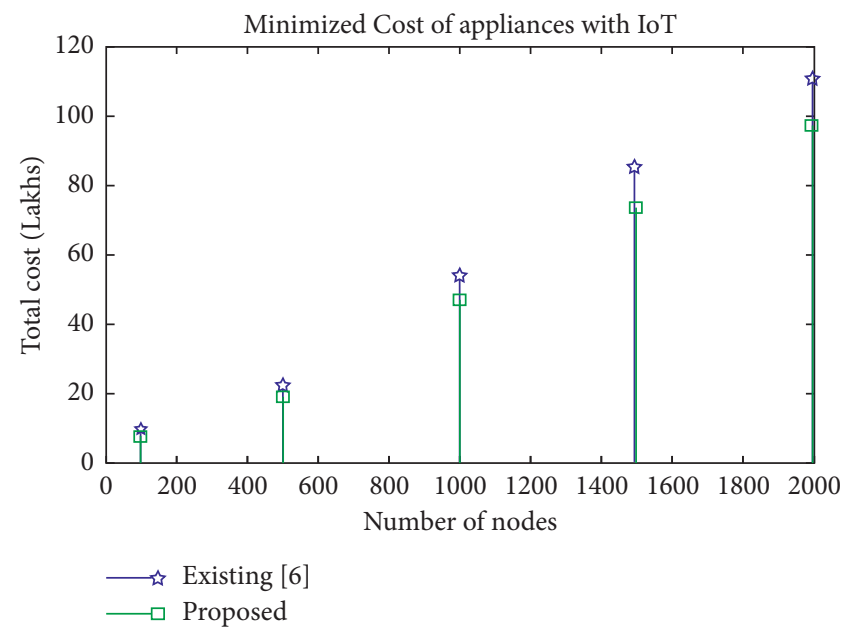

Figure 8: Total cost with a number of nodes.

observed only due to the lack of high efficient sensing devices, and combination restrictions are present. If the same method has been replaced with sensing devices, then the error in variation is observed to be less than $60 \%$ than the existing method [4]. For example, for time index, 600 seconds, the variation in signal rate is detected as 3,500 decibels, whereas with same time index, the proposed method in presence of IoT process produces the error rate of 2,300 decibels. This proves that in real-time applications more amount of energy can be saved with a low error rate that is present in the signal.

\section{Conclusion}

A new flanged intellectual device for monitoring all the appliances in households has been addressed in this article. It is tranquil to implement such intellectual devices to all appliances by dividing them into three different categories. In addition, the cost of installation will be much lesser with low energy consumption. Also, the delay of information to all 
networks will be reduced by using a dynamic management protocol. Even the hidden layers will be equipped with intellectual devices, thus forming a strong cluster region using BCANN. Moreover, the amount of charges in case of both failure and excess flow to each appliance will be certainly monitored under two different case studies. All parametric values will be observed and simulated under four different scenarios, where in all scenarios, the proposed method proves to be much efficient in terms of cost, delay, and energy consumption. In the future, the work can be extended to all commercial shops with manual protocol implementation for monitoring the flow of charges.

\section{Data Availability}

The data used to support the findings of this study are available from the corresponding author upon request.

\section{Conflicts of Interest}

The authors declare that they have no conflicts of interest.

\section{References}

[1] T. Karthick, S. Charles Raja, J. Jeslin Drusila Nesamalar, and K. Chandrasekaran, "Design of IoT based smart compact energy meter for monitoring and controlling the usage of energy and power quality issues with demand side management for a commercial building," Sustainable Energy, Grids and Networks, vol. 26, Article ID 100454, 2021.

[2] V. Kuthadi, R. Selvaraj, S. Baskar, P. Mohamed Shakeel, and A. Ranjan, "Optimized energy management model on data distributing framework of wireless sensor network in IoT system," Wireless Personal Communications, vol. 10, no. 12, 2021.

[3] M. Zekić-Sušac, S. Mitrović, and A. Has, "Machine learning based system for managing energy efficiency of public sector as an approach towards smart cities," International Journal of Information Management, vol. 20, no. 11, 2020.

[4] M. Dell'Isola, G. Ficco, L. Canale, B. I. Palella, and G. Puglisi, "An IoT integrated tool to enhance user awareness on energy consumption in residential buildings," Atmosphere, vol. 10, no. 12, 2019.

[5] K. Luechaphonthara and A. Vijayalakshmi, "IOT based application for monitoring electricity power consumption in home appliances," International Journal of Electrical and Computer Engineering, vol. 9, no. 6, pp. 4988-4992, 2019.

[6] G. Bedi, G. K. Venayagamoorthy, R. Singh, R. R. Brooks, and K.-C. Wang, "Review of internet of things (IoT) in electric power and energy systems," IEEE Internet of Things Journal, vol. 5, no. 2, pp. 847-870, 2018.

[7] D. Despa, G. F. Nama, M. A. Muhammad, and K. Anwar, "The implementation internet of things (IoT) technology in real time monitoring of electrical quantities," IOP Conference Series: Materials Science and Engineering, vol. 335, no. 1, 2018.

[8] N. H. Motlagh, M. Mohammadrezaei, J. Hunt, and B. Zakeri, "Internet of things (IoT) and the energy sector," Energies, vol. 13, no. 2, pp. 1-27, 2020.

[9] M. P. Raju and A. J. Laxmi, "IOT based online load forecasting using machine learning algorithms," Procedia Computer Science, vol. 171, pp. 551-560, 2019.

[10] G. Hafeez, Z. Wadud, I. U. Khan et al., "Efficient energy management of IoT-enabled smart homes under price-based demand response program in smart grid," Sensors, vol. 20, no. $11,2020$.

[11] M. Ozturk, M. Jaber, and M. A. Imran, "Energy-aware smart connectivity for IoT networks: enabling smart ports," Wireless Communications and Mobile Computing, vol. 2018, Article ID 5379326, 11 pages, 2018.

[12] Y. Cheddadi, H. Cheddadi, F. Cheddadi, F. Errahimi, and N. Es-sbai, "Design and implementation of an intelligent lowcost IoT solution for energy monitoring of photovoltaic stations," SN Applied Sciences, vol. 2, no. 7, 2020.

[13] P. Vengatesh Ramamurthi, E. Rajan Samuel Nadar, and E. R. Samuel Nadar, "IoT-based energy monitoring and controlling of an optimum inclination angle of the solar panels," IETE Journal of Research, vol. 17, pp. 1-11, 2020.

[14] M. Miguel, J. Boal, and Á. Sánchez-Miralles, "A literature review of IoT energy platforms aimed at end users," Computer Networks, vol. 171, 2020.

[15] S. Hanif, T. Ilyas, and M. Zeeshan, "Intrusion detection in IoT using artificial neural networks on UNSW-15 dataset," in Proceedings of the HONET-ICT 2019-IEEE 16th International Conference on Smart Cities: Improving Quality of Life Using ICT, Charlotte, NC, USA, 2019.

[16] V. Puri, S. Jha, R. Kumar et al., "A hybrid artificial intelligence and internet of things model for generation of renewable resource of energy," IEEE Access, vol. 7, pp. 111181-111191, 2019.

[17] S. Y. Hashemi and F. Shams Aliee, "Fuzzy, dynamic and trust based routing protocol for IoT," Journal of Network and Systems Management, vol. 28, 2020. 